\title{
Rapidly Growing Pulmonary Metastasis from Anaplastic Meningioma with Lethal Outcome: A Case Report
}

\author{
Marco V. Corniola ${ }^{1}$ Basile N. Landis ${ }^{2}$ Denis Migliorini ${ }^{3}$ Johannes A. Lobrinus ${ }^{4}$ Carmen Ares ${ }^{5}$ \\ Karl Schaller ${ }^{1}$ Max Jägersberg ${ }^{1}$
}

1 Service de Neurochirurgie, Département des Neurosciences Cliniques, Hôpitaux Universitaires de Genève, Genève, Switzerland

2 Service d'ORL, Département des Neurosciences Cliniques, Hôpitaux Universitaires de Genève, Genève, Switzerland

3 Service d'Oncologie, Hôpitaux Universitaires de Genève, Genève, Switzerland

${ }^{4}$ Service de Pathologie Clinique, Hôpitaux Universitaires de Genève, Genève, Switzerland

${ }^{5}$ Service de Radio-Oncologie, Hôpitaux Universitaires de Genève,

Genève, Switzerland

\begin{abstract}
Address for correspondence Dr. Marco V. Corniola, Service de Neurochirurgie, Département des Neurosciences Cliniques, Hôpitaux Universitaires de Genève, Rue Gabrielle Perret-Gentil 4, 1205 Genève, Switzerland (e-mail: Marco.Corniola@hcuge.ch).
\end{abstract}

J Neurol Surg Rep 2017;78:e129-e134.

\author{
Abstract \\ Keywords \\ - anaplastic \\ meningioma \\ - endoscopic surgery \\ - metastatic \\ meningioma \\ - skull base surgery \\ - oncology
}

\begin{abstract}
Anaplastic meningioma is seldom encountered. Moreover, distant metastasis is extremely rare, with only a handful cases reported. Here, we report the case of a 74-year-old female patient who underwent a combined cranial and endonasal approach for an extensive spheno-orbital anaplastic meningioma (WHO grade III), followed by adjuvant radiotherapy. Although local tumor control was achieved, she presented with lung metastasis 2 years later. The patient then died from pulmonary complications related to chest metastasis.

On the basis of this case, we discuss the available literature on metastatic meningiomas and radiologic follow-up strategies.
\end{abstract}

\section{Introduction}

The majority of meningiomas are benign lesions (World Health Organization [WHO] grade I) presenting with no or slow growth; watchful scan and wait strategy together with surgical excision are the standard of care for lesions that either grow or are located at unsuitable places. There is commonly no need for the evaluation of metastasis, ${ }^{1}$ and complete resection according to the Simpson classification is usually sufficient and curative but not always possible. ${ }^{2}$ In cases with more aggressive histopathologic features (WHO grade II or III), either adjuvant radiotherapy or radiosurgery is required to achieve tumor control. ${ }^{3}$ Thus, staging and radiologic follow-up are usually restricted to the local site, unless genetically inherited conditions (e.g., neurofibromatosis) are present or suspected.

Here, we present a very unusual case in which local tumor control of an extensive anaplastic spheno-orbital meningioma (WHO grade III) was achieved by combined open cranial and endonasal surgical approaches, followed by adjuvant radiotherapy. Follow-up was uneventful for 2 years up to the moment where multiple and extensive chest metastases were discovered due to newly appeared respiratory symptoms. Here, we describe the complex surgical approach applied in this case, and then the available literature on metastatic meningiomas and the potential need for extensive staging and follow-up for patients with high-grade meningiomas is discussed. received

August 8, 2016 accepted after revision

November 11, 2017
DOI https://doi.org/

10.1055/s-0037-1615808. ISSN 2193-6358.
๑) 2017 Georg Thieme Verlag KG
Stuttgart · New York

License terms

((1) $\Theta \circledast$ 


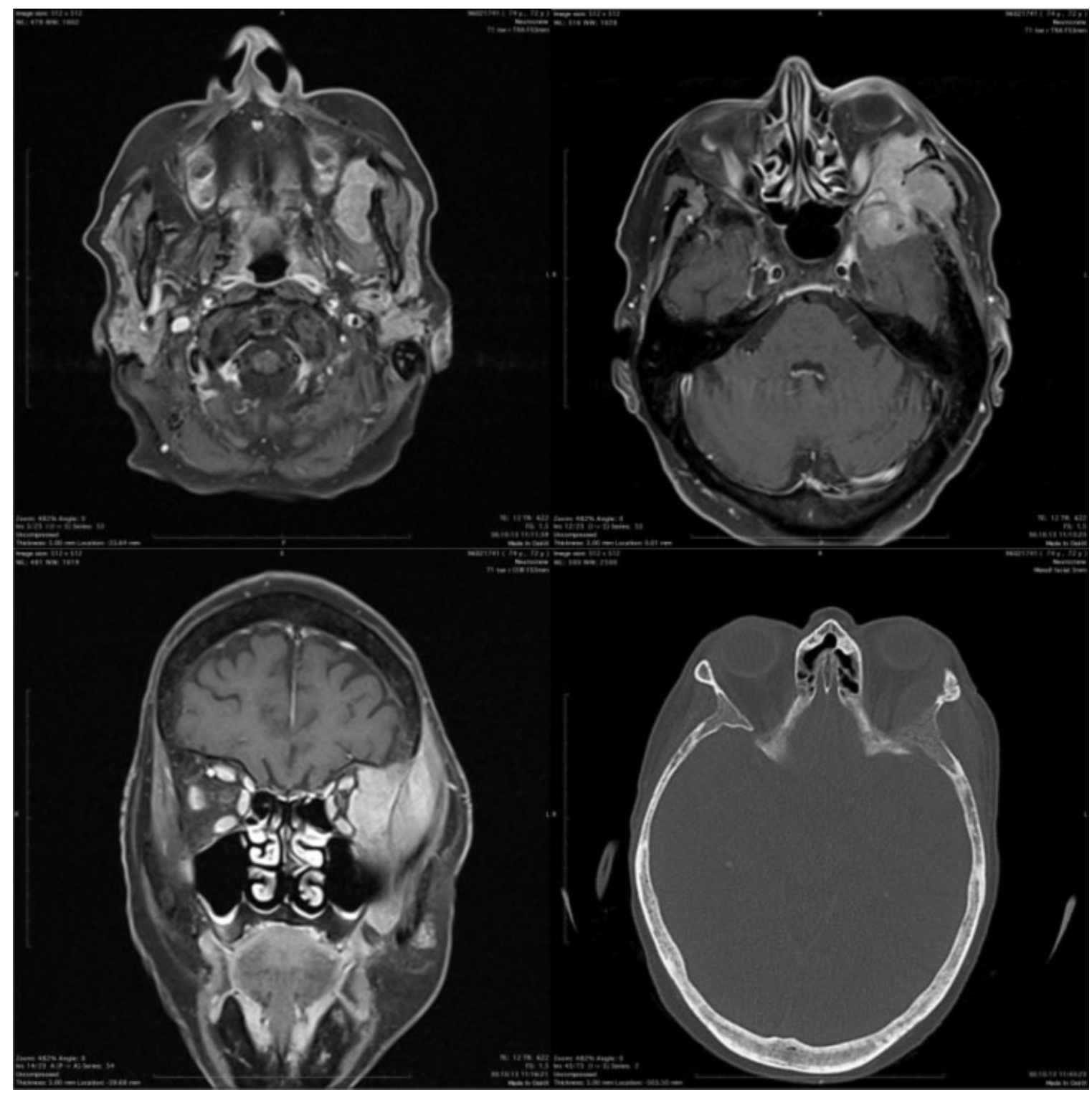

Fig. 1 Cerebral MRI showing a left contrast-enhancing mass, centered on the sphenoid bone, with inferior extension to the infratemporal fossa (A), posterior extension to the middle fossa, exerting a compression on the left temporal lobe (B), invasion of the lateral wall of the left orbit medially and the temporal muscle laterally (C), and (D) bony erosion of the left sphenoid bone.

\section{Case Description}

\section{Background and Brief Medical History}

A 74-year-old female patient with a history of chronic smoking (30 pack years) and alcohol abuse was referred to our institution with a rapidly progressing painless swelling of the left orbitotemporal region and exophthalmia without fever. Neurologic and ophthalmologic examination revealed painless limitation of abduction and elevation of the left eye, normal vision, and visual fields of both eyes. Blood samples did not show any abnormalities. Cranial CT scan and MRI showed an extensive, contrast-enhancing lesion of the left sphenoidal region with bony erosion, extending to the orbit, to the pterygopalatine fossa, the temporal fossa, and extracranially to the temporal muscle ( - Fig. 1 ). A thoraco-abdominopelvic (TAP) CT scan, which was performed prior to the referral to our clinic, did not show relevant abnormalities. Radiologic diag- nosis was in favor of meningioma. The differential diagnosis of primary central nervous system lymphoma (PCNSL) with potentially different therapeutic consequences could not be excluded at this stage; thus an open biopsy was performed first. This biopsy was performed in the temporal muscle without complications. Histologic examination showed an infiltrative tumor, with typical meningeal aspect (fascicular proliferation with whorls), but also areas of a clearly malignant component, with sheeting, large atypical cells, prominent nucleoli, and numerous mitotic figures ( $>20$ per 10 highpower field), without necrosis. The tumor cells were not only positive for epithelial membrane antigen (EMA) but also for pan-cytokeratin (clones 5B3 and AE1/AE3). $\mathrm{K}_{\mathrm{i}}-67$ proliferation index was $>20 \%$. Progesterone receptors were negative. Based on the typical meningeal aspect combined with malignant features, a diagnosis of an anaplastic meningioma, WHO grade III, was made (-Fig. $\mathbf{2}$ ). 


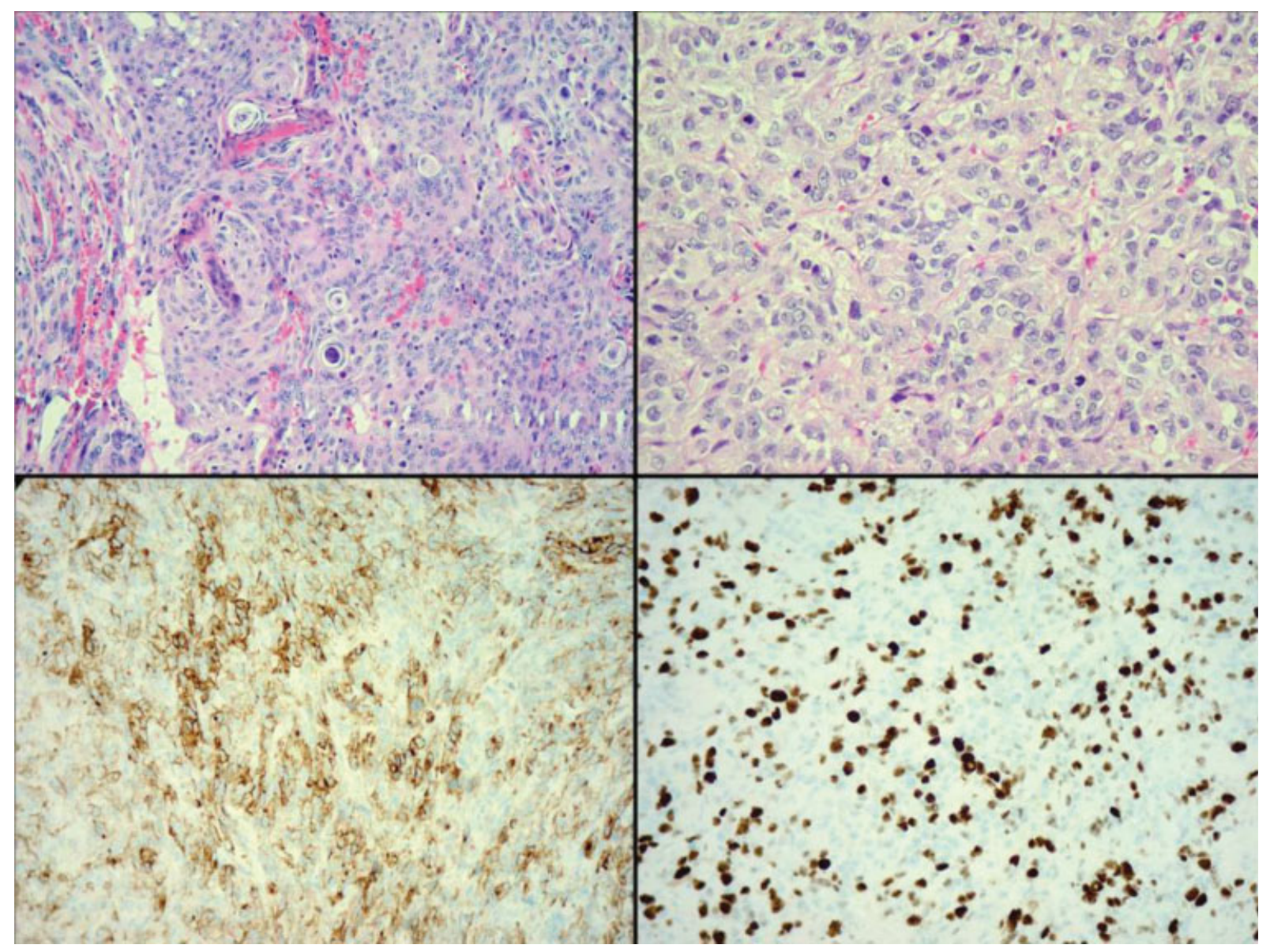

Fig. 2 Histology of the primary tumor. Upper left shows typical meningeal morphology, with whorls (Hemoatoxylin/Eosin [HE] 200x). Upper right shows malignant morphology, with sheeting, large atypical cells, and numerous mitosis (HE 400x). Lower left shows positive EMA immunostaining (200x). Lower right shows proliferation index ( $\mathrm{K}_{\mathrm{i}}-67$ immunostaining 200x).

\section{Surgical and Radiotherapeutic Management}

The patient was scheduled to undergo surgery in two distinct steps. First, a pterional craniotomy with resection of the intracranial meningioma parts and dural attachments was done. In the same time, the resection of the intraorbital and extracranial temporal and infratemporal tumor extensions was performed. This was assisted by navigation and intraoperative neuromonitoring (somatosensory evoked potentials [SSEP], motor evoked potentials [MEP] and visual evoked potentials [VEP]). No complication was observed apart from anesthesia of the second branch of the trigeminal nerve $\left(\mathrm{V}_{\mathrm{II}}\right)$ and the moderate postoperative aesthetic deformation of the lateral orbital wall. Second, 1 month later, the remaining part of the meningioma in the pterygopalatine fossa was resected with an endoscopic technique via medial maxillectomy, including reconstruction of the posterior wall of the maxillary sinus with an inferior turbinate flap (BL). Postoperative MRI and CT scan (-Fig. 3) showed no soft tumor remnant of the meningioma. Because of difficult visual conditions in the extra-cranial soft tissue and the unavoidable bony remnants (due to the location of the tumor) of the meningioma, the resection was classified as Simpson 4. Because of the malignant nature of the lesion (anaplastic meningioma, WHO grade III), an adjuvant radio- therapy was decided by the interdisciplinary tumor board ( $60 \mathrm{~Gy}$, distributed in 30 sessions of $2 \mathrm{~Gy}$ each) and was performed without complications.

\section{Radiologic Follow-Up and Clinical Evolution}

This patient was followed up for a period of 2 years. Her only complaints were general fatigue and dry nose and mouth, as a probable side effect of the radiotherapy. Radiologic followup with head MRI at 6,12, and 18 months after treatment did not show any recurrence of the spheno-orbital meningioma. No TAP imaging was performed for follow-up.

Two years after the initial diagnosis, the patient suffered a fast deteriorating dyspnea requiring her hospitalization. Thoracic CT discovered a mass of $3 \times 3.5 \mathrm{~cm}$ of the right inferior pulmonary lobe and a large right pleural effusion. The patient underwent drainage of the effusion by means of a chest tube, followed by thoracoscopy for biopsy and pleurodesis. Histologic examination showed multiple nodules of a highly malignant process, with patternless or vaguely nodular growth. One whorl was observed. The cells were large, atypical, with prominent nucleoli and nearly 40 mitoses per 10 high-power fields organized. Immunostaining was positive for EMA and pan-cytokeratin (clones 5B3 and AE1/ AE3), focally positive for $\mathrm{D} 2-40$ and protein $\mathrm{S} 100$, negative for 

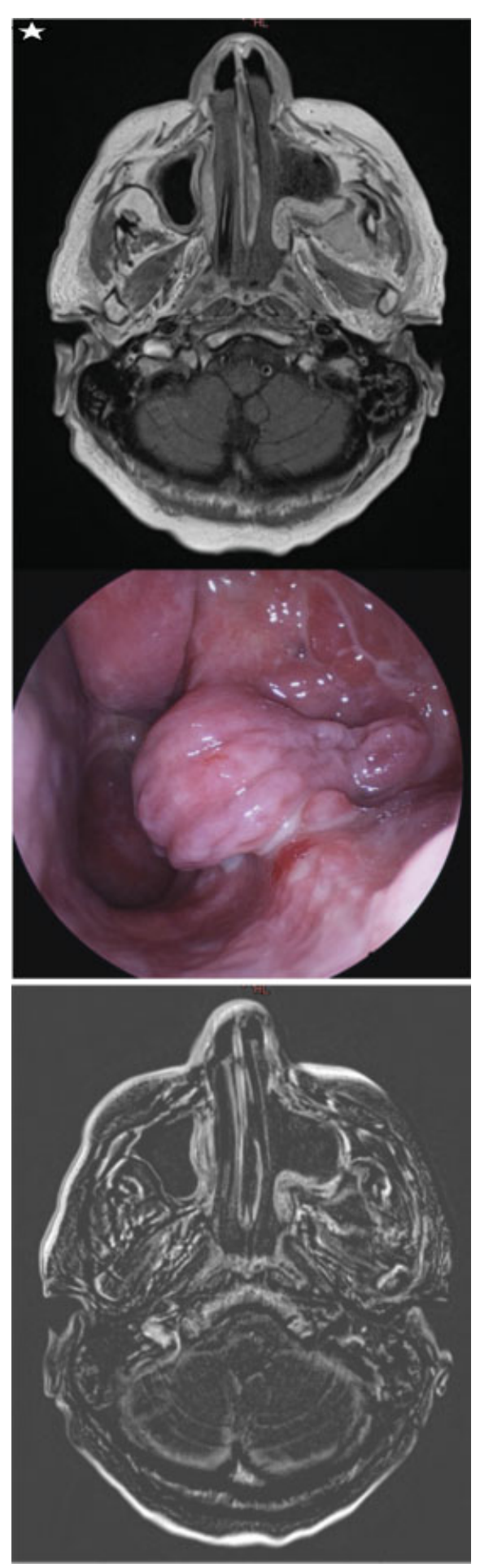

Fig. 3 (A-C)Axial postoperative cerebral MRI, showing no residual tumor in the left masticatory space. (B) (subtraction sequences) A discrete hematoma in the surgical site. The star indicates the inferior turbinate flap used to reconstruct the posterior wall of the left maxillary sinus.

TTF1, CK7, CK20, p63, BEREP4, calretinin, WT1, CD56, synaptophysin, chromogranin A, CD31, CD34, Melan-A, HMB45. $\mathrm{K}_{\mathrm{i}}-67$ proliferation index was $>40 \%$. More than 30 mitoses per 10 high-power fields were counted ( - Fig. 4 ). Given the histologic and immunohistochemical similarity between the cranial and pleural biopsies, our favored diagnosis was metastatic lesion of the known anaplastic meningioma, WHO grade III. Unfortunately, no mutation was found in the primary and metastatic tumors by next-generationsequencing (NGS) analysis (Ion Ampliseq Cancer Hotspot v2 of the Ion Proton System, with GenSearch program). The interdisciplinary tumor board scheduled chemotherapy as soon as the inflammatory response syndrome caused by the pleurodesis decreased. The patient was discharged, but returned within 3 weeks with a new respiratory decompensation and chest pain. A new thoracic CT showed a dramatic growth of the thoracic mass (-Fig. 5). Considering the fast oncologic evolution and clinical state, a palliative care was installed to comfort the patient who died 14 days later.

\section{Discussion}

Metastatic dissemination of meningiomas is a rare complication of that entity that has been described in the literature. $^{4,5}$ Two questions evolve from our case: (1) Is the pathologic diagnosis correct? (2) When should radiologic follow-up extended to other organs? Regarding the pathology, all specimens from the open biopsy, cranial surgery, and endoscopic extended approach confirmed the diagnosis of anaplastic meningioma WHO grade III. The biopsy of the thoracic mass was a malignant tumor, histologically in line with an origin from the known meningioma. NGS for mutations was negative for both tumors and hence not of help to confirm or infirm metastatic origin. All specimens were sent to a pathologic reference center that supported the diagnosis of metastatic meningioma. Concerning the extension of radiologic follow-up to other organs, the radiologic diagnosis of meningioma is safe and nearly pathognomonic from MRI. Although infrequently described in the literature, metastases from meningioma are overall extremely rare (Rawat 1995, citation n.6). Besides as it has been described in the field of high-grade gliomas, circulating tumor cells have been found despite the general concept of defining gliomas as nonmetastasizing tumors. ${ }^{6,7}$ Moreover, meninges are highly vascularized and seem to be the main drainage system of lymphatics in the central nervous system $(\mathrm{CNS}){ }^{8}$ As a result, the rationale of metastases arising from meningioma exist as the tumor is virtually in contact with the systemic blood stream. Hence, extension of radiologic screening is usually not performed unless hereditary genetic abnormalities are known or suspected. Follow-up protocols after meningioma resection differ from center to center. In our institution, MRI of the surgical site is performed at 3 months, 12 months, 2 years, and 5 years following treatment for low-risk patients (WHO grade I, Simpson 1, 2, 3) and at 3 months, 6 months, 12 months, and then once per year for all high-risk patients (WHO grade II or III with or without adjuvant radiotherapy, Simpson 2, 3, 4, 5; WHO grade I Simpson 4, 5).

\section{Extension of Radiologic Follow-Up to Other Organs}

In the presented case, a TAP CT screening had already been performed and was negative. This screening result did not 


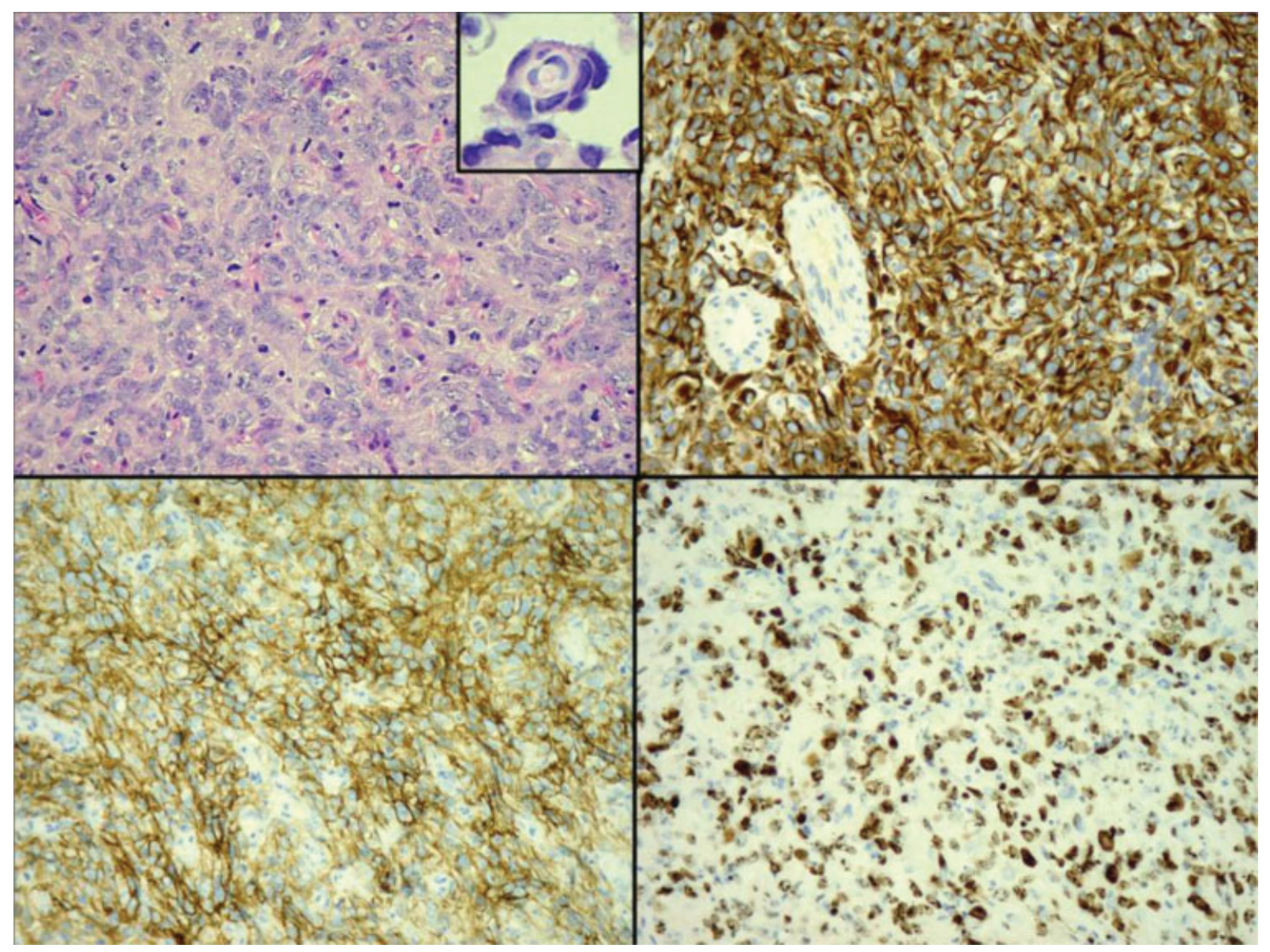

Fig. 4 Histology of the pleural metastatic lesion. Upper left shows highly atypical large cells with numerous mitosis and patternless growth (HE 200x). Inset shows a whorl (HE 600x). Upper right shows strong pan-cytokeratin positivity (immunostaining 200x). Lower left shows EMA positivity (immunostaining 200x). Lower right shows proliferation index ( $\mathrm{K}_{\mathrm{i}}-67$ immunostaining 200x).

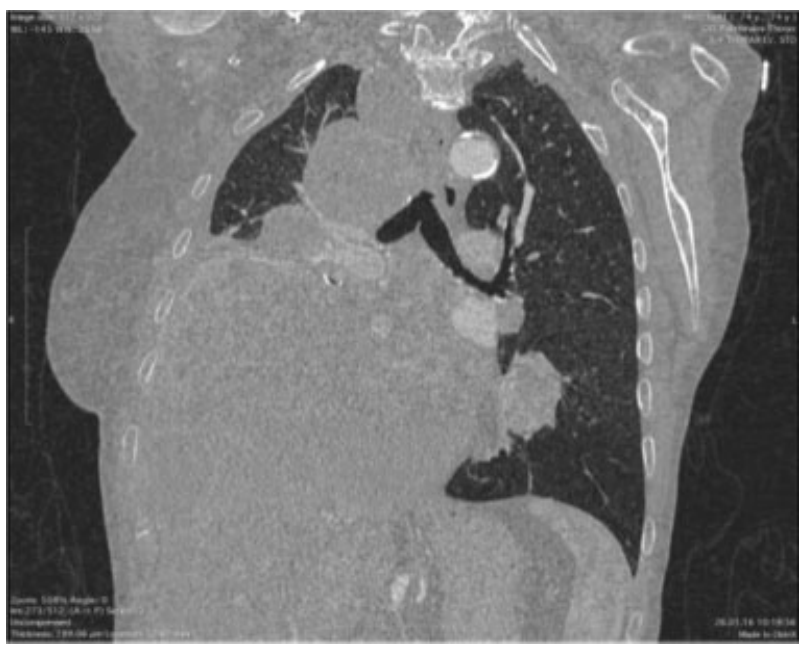

Fig. 5 Right inferior lobe (RIL) mass associated with RIL atelectasis. Another mass is found in the apical segment of the LIL. An important right pleural effusion was also present (not shown).

prevent the patient from fatal tumor spread, although local tumor control had been achieved. The available data about metastases from meningioma indicate an incidence of 1 in 1000 meningiomas. ${ }^{1,9-11}$ Anaplastic meningiomas are observed only slightly more often in this context (40\% grade III versus $33.9 \%$ grade I), but this has to be balanced with the much rarer incidence of high-grade meningioma (80-90\% grade I versus $1-3 \%$ grade III). Hence, on the basis of these figures, grade III meningiomas have a 33 -fold risk to spread compared with other meningiomas, with a relative incidence of $43 \%{ }^{4}$ To our knowledge no guidelines to radiologic follow-up for metastases in high-grade meningiomas exist. Having experienced the presented case and considering the data mentioned above, we believe that systemic imaging workup and follow-up should be discussed for patients with anaplastic meningiomas and we have decided that all our future patients with this diagnosis will receive TAP CT scan for follow-up.

\section{Conclusion}

In our opinion, anaplastic meningioma WHO grade III should be treated and followed as organic cancers, as this pathology presents a potential to spread at distant sites. When anaplastic meningioma WHO grade III is encountered, the necessity of systematically TAP CT should be considered.

\section{Disclosure}

Authors do not report any conflict of interest. 
e134 Rapidly Growing Pulmonary Metastasis from Anaplastic Meningioma with Lethal Outcome Corniola et al.

\section{References}

1 Claus EB, Bondy ML, Schildkraut JM, Wiemels JL, Wrensch M, Black PM. Epidemiology of intracranial meningioma. Neurosurgery 2005;57(06):1088-1095, discussion 1088-1095

2 Simpson D. The recurrence of intracranial meningiomas after surgical treatment. J Neurol Neurosurg Psychiatry 1957;20(01):22-39

3 Drummond KJ, Bittar RG, Fearnside MR. Metastatic atypical meningioma: case report and review of the literature. J Clin Neurosci 2000;7(01):69-72

4 Enam SA, Abdulrauf S, Mehta B, Malik GM, Mahmood A. Metastasis in meningioma. Acta Neurochir (Wien) 1996;138(10): 1172-1177, discussion 1177-1178

5 Kanthan RSJ. Distant metastasis from meningiomas - a myth or reality? Ann Clin Pathol 2013;1(01):1001

6 Rawat B, Franchetto AA, Elavathil J. Extracranial metastases of meningioma. Neuroradiology 1995;37(01):38-41
7 Müller C, Holtschmidt J, Auer M, et al. Hematogenous dissemination of glioblastoma multiforme. Sci Transl Med 2014;6(247): 247ra101

8 Sullivan JP, Nahed BV, Madden MW, et al. Brain tumor cells in circulation are enriched for mesenchymal gene expression. Cancer Discov 2014;4(11):1299-1309

9 Louveau A, Smirnov I, Keyes TJ, et al. Corrigendum: structural and functional features of central nervous system lymphatic vessels. Nature 2016;533(7602):278

10 Figueroa BE, Quint DJ, McKeever PE, Chandler WF. Extracranial metastatic meningioma. Br J Radiol 1999;72(857):513-516

11 Rampurwala M, Pazooki M, Schauer P. Delayed hepatic metastasis from a benign fibroblastic meningioma thirty-one years after surgical resection of the intracranial tumor. J Clin Oncol 2011;29 (09):e214-e215 